\section{(6) OPEN ACCESS}

\title{
The endurance athletes heart: acute stress and chronic adaptation
}

\author{
Keith George, ${ }^{1}$ Greg P Whyte, ${ }_{1}$ Danny J Green, ${ }^{1,2}$ David Oxborough, ${ }^{3}$ Rob E Shave, ${ }^{4}$ \\ David Gaze, ${ }^{5}$ John Somauroo ${ }^{1,6}$
}

${ }^{1}$ Research Institute for Sport and Exercise Sciences, Liverpool John Moores University, Liverpool, UK ${ }^{2}$ School of Sport Science, Exercise and Health, The University of Western Australia, Crawley, Western Australia, Australia

${ }^{3}$ School of Healthcare, University of Leeds, Leeds, UK ${ }^{4}$ School of Sport, Cardiff Metropolitan University, Cardiff, Wales, UK

${ }^{5}$ Department of Clinical Chemistry, St George's Hospital, Tooting, London, UK

${ }^{6}$ Cardiorespiratory and Vascular Department, Countess of

Chester Hospital, Chester, UK

\section{Correspondence to}

Dr Keith George, Research

Institute for Sport and Exercise

Sciences, Liverpool John

Moores University, Tom Reilly

Building, Byrom Street,

Liverpool L3 3AF, UK;

k.george@ljmu.ac.uk

Accepted 21 June 2012

\begin{abstract}
The impact of endurance exercise training on the heart has received significant research and clinical attention for well over a century. Despite this, many issues remain controversial and clinical interpretation can be complex of biomarkers of cardiomyocyte insult. This review assesses the current state of knowledge related to two areas of research where problems with clinical decision making may arise: (1) the impact of chronic endurance exercise training on cardiac structure, function and electrical activity to the point where the athletic heart phenotype may be similar to the expression of some cardiac pathologies (a diagnostic dilemma referred to as the 'greyzone') and (2) the impact of acute bouts of prolonged exercise on cardiac function and the presentation of biomarkers and cardiomyocyte insult in the circulatory system. The combination of acute endurance exercise stress on the heart and prolonged periods of training are considered together in the final section.
\end{abstract}

\section{INTRODUCTION}

Endurance athletes perform significant volumes of exercise training. This training places a substantial demand on the heart that acts as a physiological and metabolic stimulus for adaptation in cardiac muscle. The clinical relevance of cardiac changes with endurance exercise can be reviewed in three broad areas: (1) how does heart structure, function or electrical activity adapt to endurance training? (2) Are there any consequences for cardiac function and cardiomyocyte integrity that arise from undertaking acute bouts of (ultra)endurance exercise? (3) What, if any, clinically relevant cardiac changes can be observed in endurance athletes if lifelong endurance exercise can be documented?

These questions are relevant and are reflected in recent case studies ${ }^{1}$ and case-control series ${ }^{2}$ as well as reviews. ${ }^{3-5}$ The following review seeks to summarise and clinically contextualise the historical database, emerging data, controversies and clinical quandaries as well as to direct future research.

\section{CHRONIC ENDURANCE EXERCISE TRAINING AND THE HEART}

The clinical value of data pertaining to cardiac adaptation to chronic endurance exercise training has largely been focused on two specific ideas: (1) cardiac adaptation to training is specific to the training stimulus and (2) knowledge of the "upper limits' of physiological cardiac adaptation is vital to inform the differentiation of the athlete's heart from pathologies that may predispose the athletes to sudden cardiac death. ${ }^{6}$ Although a significant body of knowledge has been produced to address these issues, clinical uncertainty can still arise and new data are being produced.

\section{The 'Morganroth Hypothesis' and left ventricular adaptations to endurance training}

The major impetus in this field was provided by the first study to use echocardiography to image the athlete's heart. ${ }^{7}$ Morganroth and colleagues ${ }^{7}$ described an eccentric left ventricular (LV) hypertrophy in endurance athletes that reflected an increased LV internal dimension and mass, with minor changes in LV wall thickness. In a parallel group of resistance-trained athletes LV wall thickness and mass were increased but LV dimension was not, consequently the LV wall to chamber ratio was increased and Morganroth et al termed this concentric hypertrophy. A differential haemodynamic stimulus was proposed as the mechanism to explain this dichotomy and confirmatory crosssectiona $1^{8}$ and longitudinal evidence ${ }^{9}$ has prompted widespread adoption of these ideas. ${ }^{10}$ While most research has confirmed an eccentric LV hypertrophy in endurance athletes, the support for a concentric LV hypertrophy in resistance-trained athletes has been challenged by cross-sectional ${ }^{11} 12$ and longitudinal ${ }^{13} 14$ data sets.

The robust evidence supporting an eccentric LV hypertrophy in endurance athletes is exemplified by the outcome of two meta-analyses. ${ }^{15} 16$ The contention is that the LV adaptation to prolonged periods of training is important in the development of an enhanced cardio-respiratory capacity and endurance performance. Current knowledge of the upper limits of physiological adaptation of the LV is derived mainly from endurance athletes. ${ }^{17} 18$ Although more extreme LV dimensions have been reported in some endurance athletes, ${ }^{19}{ }^{20}$ these data have not been replicated in more recent studies of similar athletes. ${ }^{21-23}$ Consequently, the current consensus is that the upper limit of physiological cardiac adaptation in endurance athletes is represented by an internal dimension of the LV of $<65 \mathrm{~mm}$ and LV wall thickness of $<14 \mathrm{~mm}$ (table 1). Concern for pathology and follow-up is needed when LV dimensions exceed these data. ${ }^{23}$

Cardiac dimensions in endurance athletes are subject to significant between-subject variability, but most subjects present with values below the normative limits reported in table 1. There are, however, a small number of endurance athletes who express cardiac dimensions above these upper normal limits and this overlaps with lower levels of disease penetrance in pathologies such as dilated cardiomyopathy and hypertrophic cardiomyopathy (HCM). This overlap between physiology and 
Table 1 Normative athlete data for key LV, RV and left atrium dimensions including selected references since Pluim et al's meta-analysis in 2000

\begin{tabular}{|c|c|c|c|c|c|c|c|c|c|c|}
\hline Citation & Athletes (n) & LVM (g) & IVSd (mm) & LVDd (mm) & LVPWd (mm) & RVI (mm) & $\begin{array}{l}\text { RVOT } \\
\text { (mm) }\end{array}$ & $\begin{array}{l}\text { LAD } \\
\text { (mm) }\end{array}$ & LAvol (ml) & $\begin{array}{l}\text { LAvoll } \\
\left(\mathrm{ml} / \mathrm{m}^{2}\right)\end{array}$ \\
\hline La Gerche $^{24}$ & Cyclists/runners (40) & & $11.0 \pm 1.5$ & $56 \pm 5$ & & & & & & \\
\hline George $^{21}$ & $\begin{array}{l}\text { Ultramarathoners } \\
(\mathrm{M}=126, \mathrm{~F}=39)\end{array}$ & $\begin{array}{l}\text { M: } 193 \pm 42 \\
(106-300) \\
\text { F: } 134 \pm 25 \\
(89-186)\end{array}$ & $\begin{array}{l}\text { M: } 11.0 \pm 2.0 \\
(8.0-14.0) \\
\text { F: } 10.0 \pm 1.0 \\
(6.0-12.0)\end{array}$ & $\begin{array}{l}\text { M: } 53 \pm 4 \\
(46-62) \\
\text { F: } 49 \pm 3 \\
(42-55)\end{array}$ & $\begin{array}{l}\text { M: } 10.0 \pm 1.0 \\
(7.0-12.0) \\
\text { F: } 8.0 \pm 1.0 \\
(6.0-10.0)\end{array}$ & & & $\begin{array}{l}37 \pm 4 \\
(25-47)\end{array}$ & & \\
\hline Wilhelm ${ }^{27}$ & Runners $(\mathrm{M}=60, \mathrm{~F}=61)$ & & $\begin{array}{l}\text { M: } 11.2 \pm 1.1 \\
\text { F: } 9.2 \pm 1.1\end{array}$ & & $\begin{array}{l}\text { M: } 10.7 \pm 1.0 \\
\text { F: } 9.2 \pm 1.3\end{array}$ & & & & $\begin{array}{l}\text { M: } 56 \pm 6 \\
\text { F: } 50 \pm 3\end{array}$ & $\begin{array}{l}\text { M: } 29 \pm 7 \\
\text { F: } 30 \pm 6\end{array}$ \\
\hline Abergel $^{23}$ & Cyclists (286) & & $11 \pm 1.3$ & $60 \pm 4$ & $10.0 \pm 1.0$ & & & & & \\
\hline Nagashima $^{19}$ & Ultramarathoners (291) & & $\begin{array}{l}10.2 \pm 1.9 \\
(5.0-19.0)\end{array}$ & $\begin{array}{l}62 \pm 7 \\
(42-75)\end{array}$ & $\begin{array}{l}10.0 \pm 1.4 \\
(5.0-15.0)\end{array}$ & & & $\begin{array}{l}40 \pm 5 \\
(26-49)\end{array}$ & & \\
\hline
\end{tabular}

Data are mean $\pm S D$ and (range) where applicable.

LVM, left ventricular mass; IVSd, interventricular septal thickness at end-diastole; LVDd, LV internal dimension at end-diastole; LVPWd, LV posterior wall thickness at end-diastole; RVI, right ventricular inflow dimension; RVOT, RV outflow tract dimension; LAD, left atrial diameter; LAVol, LA volume; LAVoll, LAVol index; M, males; F, females; LV, left ventricle; $\mathrm{RV}$, right ventricle; LA, left atrium.

pathology has been termed the 'grey-zone'28 and reflects an area of diagnostic uncertainty. Approximately $80 \%$ of nontraumatic sudden deaths in young athletes are caused by inherited or congenital cardiac defects of which HCM is the most common pathology associated with sudden cardiac death. ${ }^{29}$ Superior athletic performance can co-exist with a hereditary cardiac disease; however, athlete deaths where HCM is implicated predominantly occur in intermittent power/speed sports such as soccer, American football and basketball. ${ }^{30}$ The observation of HCM in endurance athletes is rare, ${ }^{30}{ }^{31}$ likely because HCM and an enhanced cardiac output sufficient to underpin endurance performance are thought to be incompatible.

Like cardiac morphology, there are some ECG patterns that have been reported in both the endurance athlete's heart and cardiac pathologies and these can overlap in the 'grey zone'. Common ECG findings in the endurance athlete's heart, related to training, include sinus bradycardia, sinus arrhythmia, conduction delays, early repolarisation of the ST segment and isolated voltage criteria for LV hypertrophy (termed Group 1 ECG changes). ${ }^{32} 33$ A recent consensus statement ${ }^{33}$ also identified uncommon and usually training-unrelated ECG findings in athletes that include: T-wave inversion; ST-segment depression; pathological $\mathrm{Q}$-waves; left atrial enlargement; left axis deviation/left anterior hemiblock; right axis deviation/left posterior hemiblock; right ventricular hypertrophy; ventricular preexcitation; complete left or right bundle branch block; long-OT or short-QT interval; and Brugada-like early repolarisation (termed Group 2 ECG changes). These changes should be treated suspiciously for the presence of cardiac pathology and usually warrant further investigation. As an example, T-wave inversion $\geq 2 \mathrm{~mm}$ in two or more adjacent leads, especially in the inferior and lateral leads, is associated with risk of sudden cardiac death in cardiomyopathy (HCM or arrhythmogenic right ventricle cardiomyopathy (ARVC)), ischaemic heart disease, aortic valve disease, hypertension and LV noncompaction. ${ }^{33-37}$ These changes however may be a normal variant, especially anteriorly, with associated ST elevation preceding the T-wave inversion and when no other ECG abnormalities are present. Likewise $\mathrm{T}$-wave inversions are more common in black athletes ${ }^{38} 39$ and the clinical relevance in these groups is not fully understood. Some common ECG changes may not apply to other ethnic groups and masters' endurance athletes ([Cooper $\mathrm{R}$, et al, unpublished data). For example, in asymptomatic endurance athletes $>35$ years of age with no documented history of coronary artery disease, the resting ECG has high false-negative and false-positive rates. ${ }^{40-42}$ Half of older athletes with normal coronary arteries on angiography have ECG abnormalities. The ECG as a screening tool in this older population is limited and the European Society of Cardiology recommends self-assessment using a validated questionnaire followed by exercise testing by a physician if the systematic coronary risk evaluation shows an increased risk for coronary events. ${ }^{43}$ Further research is required in this area.

\section{Emerging evidence: the RV and left atria in the endurance athlete}

Understanding RV adaptation to endurance exercise will inform the diagnostician in the process of differentiating physiological adaptation from inherited cardiomyopathies such as ARVC, which accounts for approximately $4 \%$ of cardiac sudden death in the athletic population. ${ }^{29}$ Similar to the LV, eccentric RV hypertrophy has been documented in endurance athletes. ${ }^{44} 45$ In support of cardiac magnetic resonance studies, recent echocardiographic studies have provided a more comprehensive evaluation of RV structure ${ }^{24-26}$ and some normative data are presented in table 1. D'Andrea et $a l^{26}$ and Oxborough et $a l^{25}$ demonstrated larger RV diameters at both the RV inflow and outflow in endurance athletes compared to published normal ranges, sedentary controls and strength-trained athletes. ${ }^{26}$ Furthermore, Oxborough et al ${ }^{25}$ demonstrated an increased RV: LV ratio, suggesting that the degree of remodelling maybe unequal in endurance athletes. This could be explained by a disproportionate wall stress being applied to the thin-walled $\mathrm{RV}^{46}$

In view of ARVC being one of the main causes of cardiac sudden death in the athlete, ${ }^{29}$ these findings create a challenging diagnostic dilemma. Although the RV outflow tract in the endurance athlete is generally larger than in the normal healthy population, the RV inflow appears to be dilated to a greater degree. $^{25}$ It is predominantly the RV outflow that is enlarged in ARVC and therefore the presence of a dilated RV inflow may be 
more representative of physiological conditioning in this setting.

It is important to consider RV function in the presence of significant RV remodelling, in that ARVC often results in an impairment to RV function. ${ }^{47-49}$ The development of strain imaging (figure 1) has provided scope for quantitative evaluation of regional and global RV function ${ }^{50}$ and Teske et al ${ }^{51}$ highlighted lower RV global strain values in elite endurance athletes, owing to a reduction in basal function. This finding has been reproduced in 40 endurance athletes at rest yet with augmented apical function ${ }^{24}$ that may suggest that global RV strain is likely to be a useful indicator of physiological adaptation. ${ }^{25}$ La Gerche et $a l^{24}$ demonstrated an enhanced contractile reserve of the basal RV segment during exercise and proposed that stress echocardiography may provide additional diagnostic value.

In order to provide a sensible approach to the differentiation of ARVC from endurance athletic conditioning the presence of RV inflow dilatation, normal RV function during exercise (demonstrated using standard or quantitative echocardiography) and the lack of saccular outpouching in the RV outflow are consistent with physiological adaptation. Finally, the presence of a prominent RV moderator band has been evidenced in both ARVC $^{48}$ and the endurance athlete ${ }^{49}$ and therefore should not be used as diagnostic criteria.

The left atrium (LA) of the endurance athlete has received even less attention than the RV. Oxborough et al ${ }^{25}$ observed a large indexed LA volume, with 88\% of the 102 endurance athletes having values above the American Society of Echocardiography's normal range (figure 2 and table 1). This is also of importance when differentiating physiology from pathology and should be considered a normal finding in endurance athletes. It is difficult to fully ascertain the prognostic implications of LA remodeling; however, there is some evidence to suggest the endurance athlete has an increased risk of atrial fibrillation/flutter ${ }^{27} 52$ which correlates well with LA size. ${ }^{53}$ The LA is known to dilate in a non-uniform manner and therefore the use of LA volume and LA area is recommended over and above standard linear LA dimension ${ }^{54}$ and therefore may provide additional prognostic / diagnostic value. ${ }^{55}$ There is evidence of superior LA function in elite athletes when compared with patients with hypertensive LV hypertrophy; ${ }^{56}$ however, there are no data specifically in the endurance athlete population.

In summary, the heart of an endurance athlete is more than likely to demonstrate changes in morphology, function and electrical activity. This may place some endurance athletes in the diagnostic 'grey-zone' and it is crucial that an accurate determination of physiology or pathology is made. New research, with developing tools, is providing more insight into the phenotype of the athlete heart by detailing morphological adaptation in the RV and LA as well as documenting multiple facets of global and regional function. More data are required in heterogeneous groups of endurance athletes and in testing the utility of new imaging tools in helping diagnostic decision making.

\section{CARDIAC RESPONSES TO ACUTE (ULTRA)ENDURANCE EXERCISE}

The endurance athlete's heart is viewed as healthy, highly responsive to acute exercise and resistant to fatigue and damage. This pervasive view has been challenged by recent reports of an acute reduction in cardiac function and the release

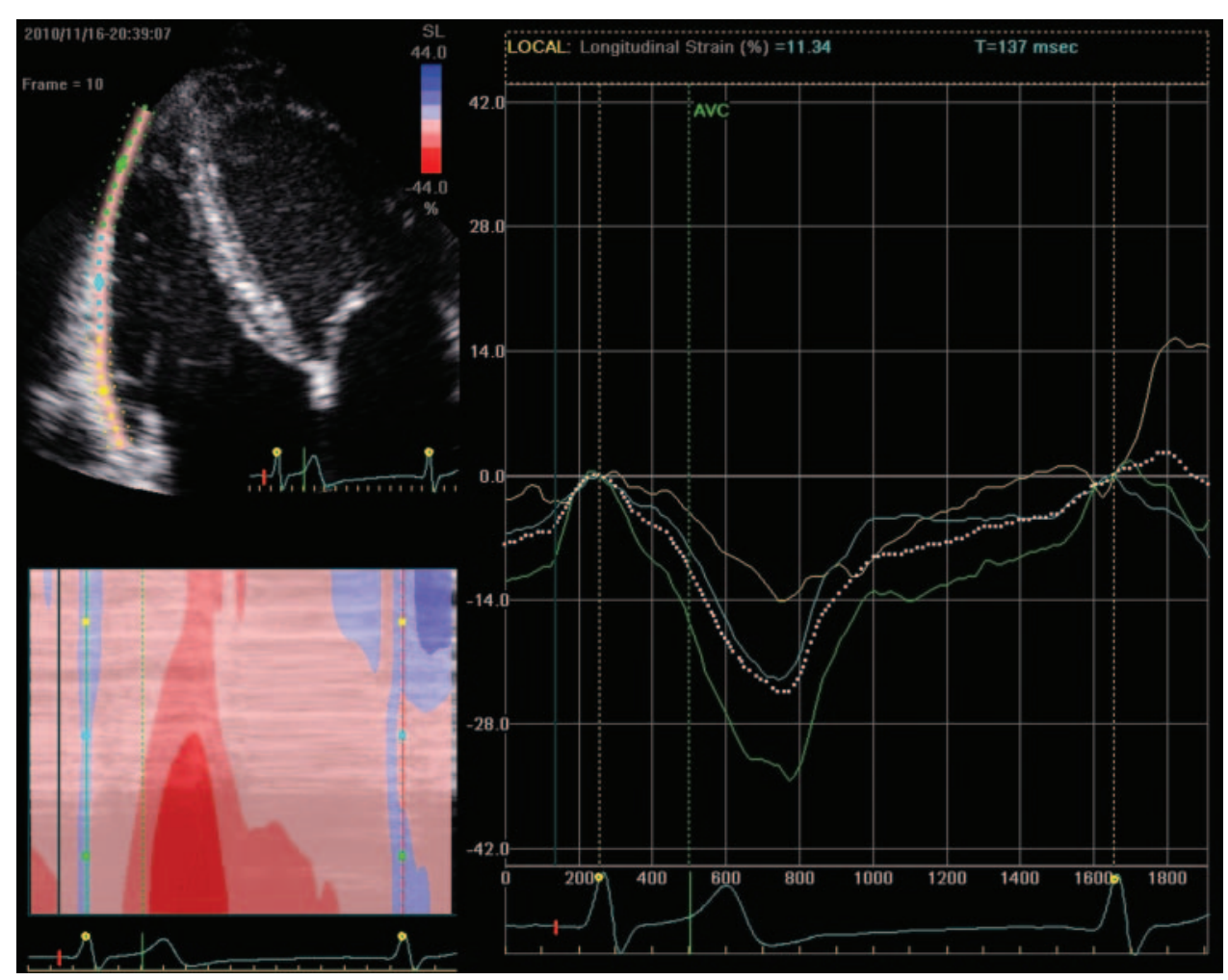

Figure 1 Exemplar two-dimensional sector scan with myocardial speckle tracking of the right ventricle free wall and longitudinal strain data presented for basal, mid wall and apical wall segments. Speckle tracking determines deformation (strain) and the rate of deformation (strain rate) is specifically targeted wall segments that are determined by a semiautomated wall tracking system. In this figure it is useful to note the higher deformation values at the apex relative to the base. 


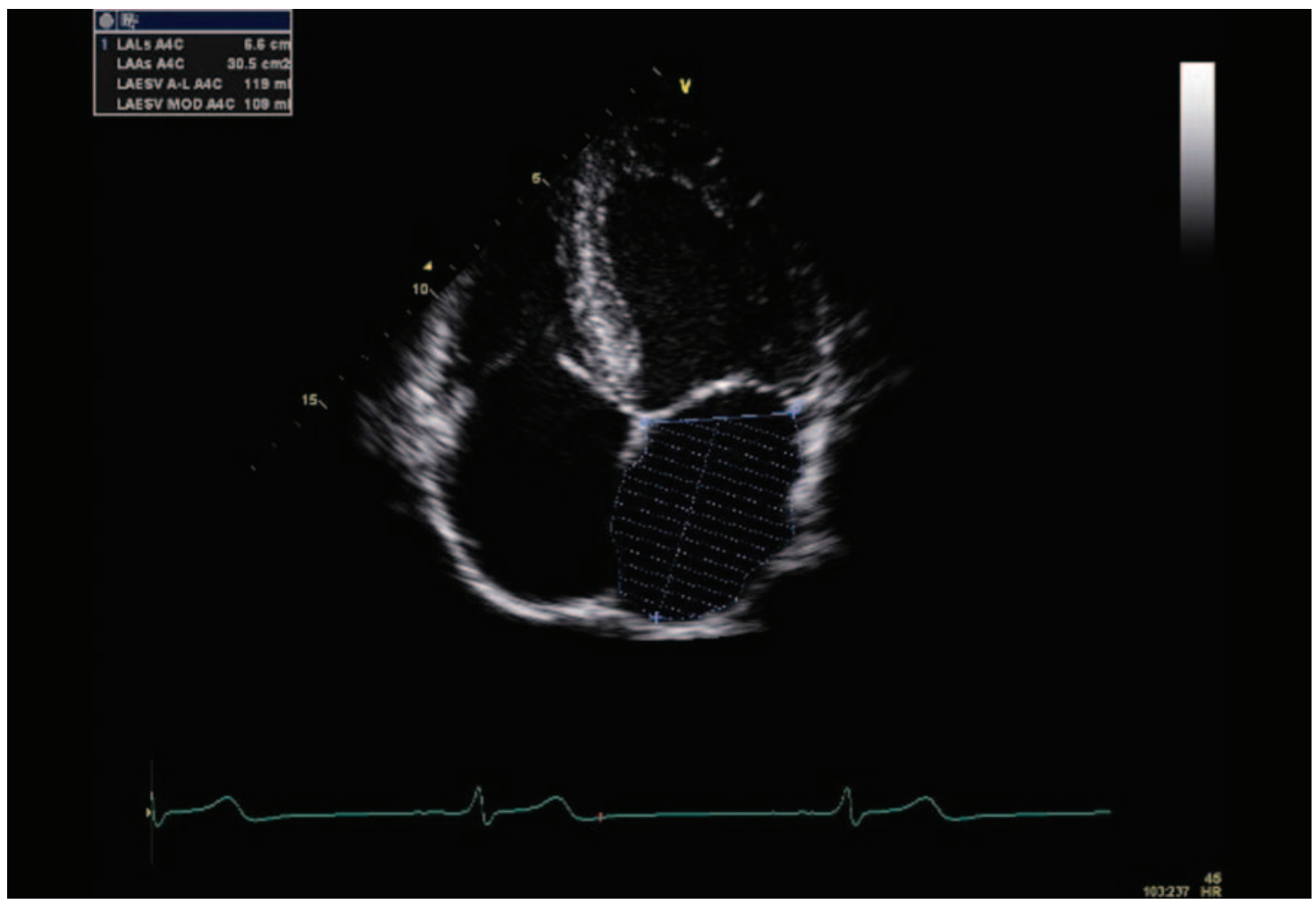

Figure 2 Exemplar two-dimensional sector scan demonstrating the assessment of LA area (and consequently volume) using the Simpsons biplane method.

of cardiac biomarkers, which are highly specific for cardiomyocyte stress or damage, in response to acute bouts of (ultra) endurance exercise.

\section{(Ultra)Endurance exercise and cardiac function}

During (ultra)endurance exercise, the total cardiac work is considerable and the heart must also cope with an elevation in core temperature, increased levels of catecholamines, increased mechanical work, altered $\mathrm{pH}$ and exposure to reactive oxygen species. ${ }^{57}$ Whether the heart can maintain performance in the face of such challenges has been the focus of recent debate. Since Saltin and Stenburg ${ }^{58}$ suggested that prolonged exercise could impair intrinsic cardiac contractile function, a number of studies have addressed the phenomenon of 'exercise-induced cardiac fatigue'. In an attempt to provide consensus our group ${ }^{59-61}$ and others ${ }^{62}$ have reviewed the literature and Middleton et al ${ }^{59}$ performed a meta-analysis, collating data related to the effect of prolonged exercise on ejection fraction (global LV contractility) and the ratio of early to atrial peak diastolic filling velocities (global LV diastolic filling). A significant overall effect of exercise was noted for both parameters. The post-exercise reduction in ejection fraction was small (c. $2 \%$ ), was mediated by increasing exercise duration and poor training status and was related to changes in ventricular dimension (an index of preload). This latter point suggests that the reduction may be attributed, in part, to altered cardiac loading and not intrinsic cardiac function per se. The post-exercise decline in diastolic filling was more consistent between studies and was independent of loading and heart rate, suggesting a direct effect of exercise upon lusitropic function.

Ongoing developments with non-invasive imaging (tissue-Doppler imaging, strain/strain rate assessment), has extended our knowledge in this area. For example, speckle- tracking assessment of myocardial deformation has allowed strain and strain rate to be assessed in longitudinal, radial and circumferential planes and at basal, mid- and apical wall levels of the LV and in the longitudinal plane for the RV and LA. Using these approaches recent work has described a negative effect of prolonged exercise on RV function ${ }^{2} 6364$ and LA function. ${ }^{65}$ Recent work by La Gerche et al ${ }^{64}$ suggests that exercise-induced changes in function may occur in the RV but not the LV. A postexercise drop in RV function was linked to cardiac biomarker appearance and increased race duration. While RV ejection fraction had returned to baseline one week after exercise, the link between a lower RV ejection fraction and chronic structural changes (evidence of cardiac fibrosis) would suggest that the clinical implications are worthy of ongoing study.

The determination of mechanism(s) underpinning changes in cardiac function following prolonged exercise is challenging, ${ }^{66}$ especially when dealing with human subjects using noninvasive imaging techniques. Although altered loading and heart rate may account for a proportion of the change in cardiac function, there is some evidence that supports other mechanisms. A downregulation of $\beta$-adrenoreceptors, related to reduced contractile state, has been shown previously in humans, ${ }^{67} 68$ although there are conflicting data in an animal model. ${ }^{69}$ Recently, Chan-Dewar and colleagues ${ }^{70}{ }^{71}$ reported an increase in the electromechanical delay in the heart after prolonged exercise that was related to a decline in peak systolic tissue velocity. This suggests that the site of cardiac fatigue is beyond the electrical activation process and thus intrinsic to the myocytes. Whether this reflects changes in energy metabolism (substrate availability) or alterations in calcium handling cannot be deduced from human studies at this point in time. Another mechanism, which has received much attention, is a direct link between cardiomyocyte damage and reduced 
function. Data related to this specific theory are presented in the next subsection.

The concept of exercise-induced cardiac fatigue remains controversial and the clinical relevance of postexercise changes in cardiac function is not fully evaluated. Most descriptive data detail a rapid recovery of $\mathrm{LV}$ and/or RV dysfunction. ${ }^{60}$ There are, however, occasional reports of more persistent changes in function. ${ }^{2}{ }^{72} \mathrm{La}$ Gerche et al ${ }^{2}$ observed a postexercise reduction in RV tissue velocities that remained depressed at 1 week in one athlete, while Neilan et l $^{72}$ reported reduced diastolic function 3-4 weeks following completion of the Boston Marathon. Further, a suggested link between increased RV end-systolic wall stress with exercise, RV dysfunction, RV remodelling and clinically relevant RV arrhythmias, in some endurance athletes, ${ }^{73} 74$ requires further study.

\section{(Ultra)Endurance exercise and cardiac biomarker release}

The potential that prolonged exercise can induce cardiomyocyte damage, which may underpin changes in heart function, has received significant attention recently. ${ }^{45}$ Multiple studies have reported significant elevations in cardiac troponin I or T (cTnI, cTnT), which are highly cardio-specific markers of cellular damage. ${ }^{4}$ These data have been reviewed ${ }^{46}$ and subjected to meta-analysis. ${ }^{77}$ In this meta-analysis, the overall event rate (positive serum sample for cTnT after prolonged exercise) was $47 \%$. Continuing studies ${ }^{78} 79$ have attempted to attribute cTn release following prolonged activity to exercise characteristics (eg, duration or intensity) or subject-related parameters (eg, age or training status). To date, these data lack consistency and generally explains only a small proportion of the variance in data. Individual studies generally assess a single blood draw postexercise. We caution against this limited analysis as this will likely underestimate the true rate of cTn appearance during or following ultraendurance events. A unique laboratory-based marathon with blood draws every 30 min during the race and at frequent intervals during recovery observed elevated cTn in all runners. ${ }^{80}$

The clinical relevance of cTn release with exercise, and whether an elevated cTn explains changes in LV and RV function, is controversial. Following exercise the concentrations of cTn released are typically very small with very rapid appearance $^{81}$ and removal. This is in contrast to cTn kinetics following myocardial infarction. ${ }^{4}$ Exercise is possibly the only documented cause of cTn release that is not associated with adverse clinical outcome. This has led to the hypothesis that an elevation in cTn is part of a normal physiological response to exercise 4 and does not represent irreversible cell death. The mechanism(s) by which cTn moves from the cardiomyocytes to the intravascular space, during and after exercise, are not known. It is possible that cTn 'leaks' from the small fraction of unbound cTn contained in the cytosol. As the physiological environment $(\mathrm{pH}$, temperature, oxidative and mechanical stress) during exercise changes, cardiomyocyte membrane permeability may also increase allowing cTn to move out of the cell. While most authors who have simultaneously assessed postexercise cardiac function and cTn release report no direct association, ${ }^{4}$ others have used correlational analysis to link the appearance of cTn to the reductions in cardiac function, notably in the $\mathrm{RV}^{2}{ }^{64}$

In summary, there is now convincing evidence that the performance of (ultra)endurance exercise can result in a small but temporary reduction in LV and RV function. At the same time, but likely due to different mechanisms, there is a release of cTn into the blood stream. It is likely that in most people these events are a normal, potentially adaptive, process associated with the 'work' of prolonged exercise. Further study is required to determine if the RV is more susceptible to 'cardiac fatigue' with prolonged exercise, whether these changes are associated with tissue damage and what the long-term consequences of such mal-adaptation might be.

\section{CLINICALLY RELEVANT ISSUES IN THE LIFELONG ENDURANCE ATHLETE}

Aerobic or endurance-based physical activity reduces cardiovascular risk. ${ }^{82}$ Epidemiological data detailing the consequence of a lifetime of endurance training on cardiovascular morbidity and mortality are limited by the fact that the global population of elite endurance athletes is relatively small and widely dispersed. Despite this, a recent epidemiological study reported a reduction in all-cause mortality in three small age-groups of male cross-country skiers followed up after 30 years in Scandinavia. ${ }^{83}$ The ability to compare cardiovascular events and deaths between these athletes and the general population was limited due to the small sample sizes studied. Consequently, generalisability is limited and further research is warranted but, clearly, there does not appear to be any 'epidemic' of cardiovascular problems in lifelong endurance athletes. Despite this, there are sporadic case reports of cardiac events in endurance athletes and there has recently been renewed interest as to whether lifelong endurance training may precipitate some pathological cardiovascular consequences in a small proportion of athletes. Specific interest has been directed towards the concept of adverse cardiac remodelling and fibrosis as well as arrhythmias and ECG abnormalities.

While endurance training can result in significant cardiac remodelling some interest has been given to whether these changes are reversible and/or can eventually lead to pathological events. It has long been known that short-term deconditioning is associated with some regression in cardiac dimensions ${ }^{84}$ but two recent studies from Italy have made significant progress in this area. Pelliccia et al ${ }^{85}$ studied 114 young Olympic endurance athletes free of cardiovascular disease over a mean of 8.6 years (range of follow-up 4-17 years). Over this period, no cardiac events or pathological diagnoses occurred and they concluded that up to 17 years of intense, uninterrupted endurance training was not associated with the development of any abnormal cardiac dimensions, any deterioration in LV function and no cardiovascular symptoms. In a different study, Pelliccia and colleagues ${ }^{86}$ prospectively followed 40 elite male athletes (mostly endurance based) with large cardiac dimensions (LVIDd $>60 \mathrm{~mm}$, wall thickness $>13 \mathrm{~mm}$ ), over a 5.6 years deconditioning period (range 1-13 years). The withdrawal from high volumes of intense training led to a reduction in cardiac dimensions at the group level. While all athletes demonstrated a reduction in wall thickness to below $13 \mathrm{~mm}$, nine athletes still had an LVIDd above $60 \mathrm{~mm}$. The authors suggested that in some athletes the resolution of cavity enlargement was incomplete and could not rule out future clinical implications. Further research tracking elite endurance athletes over longer periods of time, postretirement, seem necessary to illuminate these initial findings.

A different element of structural cardiac remodelling has been the focus of recent research in endurance athletes. The presence of myocardial fibrosis in the heart of trained subjects has been observed in case studies of endurance athletes, ${ }^{87} 88$ animals undergoing high volumes of endurance training 89 and three case series in endurance athletes. ${ }^{64} 9091$ The study of fibrosis may be important as it could provide a substrate for the increased prevalence of arrhythmias, particularly in veteran athletes. Fibrosis in the RV was recently reported in an animal model of overtraining 89 and was associated with diastolic 
dysfunction and atrial dilation. Five out of 12 rats that had undergone training had ventricular tachycardia induced compared to just one control animal. ${ }^{89}$ Interestingly, the changes were reversed after 8 weeks recovery from training. Breuckmann $e t a^{90}$ assessed the presence of interstitial cardiac fibrosis using late gadolinium enhancement on MRI scans in 102 older marathon runners, with a recent history of race completion, compared to age-matched controls. Evidence of fibrosis was observed in scans from 12 endurance athletes and 4 controls, with 7 of the athletes presenting a non-coronary artery disease pattern of fibrosis. In a smaller cohort of truly lifelong elite (ultra)endurance athletes, late gadolinium enhancement was reported in 6 of 12 athletes compared to an absence of any fibrosis in age-matched controls or younger (ultra)endurance athletes. ${ }^{91}$ Again, the pattern of fibrosis in $5 / 6$ lifelong endurance athletes was non-CAD in origin. ${ }^{91}$ In a recent study of 39 endurance athletes, late gadolinium enhancement was apparent in 5 athletes localised in the interventricular septum. ${ }^{64}$ Although no control group was studied, those athletes with evidence of fibrosis had a greater cumulative training exposure and a lower RV ejection fraction than those athletes with no fibrosis. These studies could only speculate as to the cause of such fibrosis and no long-term follow-up has been completed to determine what, if any, clinical consequence these findings may have. The aetiology and clinical significance of these structural and electrical changes remains to be fully elucidated.

As noted in the section Emerging evidence: the right ventricle and left atria in the endurance athlete, there is a growing body of evidence that masters endurance athletes have a greater prevalence of atrial flutter/fibrillation compared to non-active controls. ${ }^{52} 535592$ In a recent study, Claessen et al ${ }^{93}$ observed that a significantly high proportion of patients presenting for atrial flutter ablation surgery were regular sportsmen and concluded that a history of endurance sports and subsequent LA remodelling may be a risk factor for the development of atrial flutter. This latter point is supported by Molina et al. ${ }^{53}$ Ventricular arrhythmias have also been reported in trained athletes $^{94}$ but are normally benign, reduce with detraining, ${ }^{95}$ are independent of cardiac remodelling ${ }^{95} 96$ and appear to be suppressed after retraining. ${ }^{94}$

More complex cardiac arrhythmias have also been reported in small numbers of endurance athletes. Heidbuchel et $\mathrm{al}^{73}$ reported on a case series of 46 endurance athletes (mainly cyclists) with symptomatic arrhythmias that were largely of RV origin. Over a 5 -year follow-up, nine sudden cardiac deaths were reported. In the absence of cardiovascular disease the authors speculated that for some athletes endurance training may contribute to the development and/or progression of an underlying arrhythmogenic substrate. In a follow-up study from the same group, Ector et $a l^{74}$ noted a significantly reduced RV ejection fraction in endurance athletes with ventricular arrhythmias. They concluded that endurance exercise could act as a trigger for arrhythmias as well as contributing to changes in RV function. This group has speculated that there may be a link between the acute effects of prolonged exercise on RV function and long-term clinical complications in some endurance athletes; coining the term 'exercise-induced right ventricular cardiomyopathy'.

In summary, it is very likely that in the vast majority of endurance athletes a lifelong habit of training will improve cardiovascular morbidity and mortality. Further research is required, however, to determine the potential for detrimental cardiovascular effects of lifelong endurance exercise in a very small proportion of athletes and how these individuals may be detected and treated.

\section{CONCLUSIONS}

The heart of the endurance athlete is placed under great stress during training and competition. Cardiac adaptation to exercise training encompasses morphological, functional and electrical changes that are referred to as the 'athletic or athletes' heart'. For the most part, the endurance athletic heart is easily differentiated from pathologies that may present with similar phenotypical characteristics. For those athletes that do present in the diagnostically challenging 'grey zone', on-going study will likely further refine physiologically normative data. Acute (ultra) endurance exercise bouts represent a significant stress to the heart and there is now substantive evidence of 'cardiac fatigue' and/or biomarker release associated with prolonged activity. It is, however, entirely likely that for the vast majority of endurance athletes, the stress of acute exercise will lead to healthy, physiological adaptation in the heart. For a very small minority, though, there is emerging evidence that endurance exercise may be part of a patho-physiological cascade that clinicians must be aware of and respond appropriately too.

\section{What this review adds}

- The endurance athlete will develop morphological, functional and electrical characteristics of the athletic heart and for a small minority this will place them in a diagnostic 'grey-zone'.

- Developing techniques, such as 3D and speckle-tracking echocardiography as well as cardiac magnetic resonance, that accurately assess cardiac structure and function at a global and regional level will likely impact upon any diagnostic dilemmas.

- The cardiac work performed during endurance exercise can be profound to the point that 'cardiac fatigue' and biomarkers of cardiac damage have been reported in endurance athletes after acute exercise.

- In the vast majority of endurance athletes, the chronic accumulation of acute exercise stress will produce a healthy, physiological adaptation. In a small number, endurance exercise may be implicated in various pathological cascades that are of relevance to the athlete and their medical support team.

Contributors All authors contributed to the conception, writing and editing of the manuscript.

Competing interests None.

Provenance and peer review Commissioned; externally peer reviewed.

\section{REFERENCES}

1. Whyte G, Stephens N, Senior R, et al. Treat the patient not the blood test: the implications of an increase in cardiac troponin after prolonged endurance exercise. Br J Sports Med 2007;41:613-15.

2. La Gerche A, Connelly KA, Mooney DJ, et al. Biochemical and functional abnormalities of left and right ventricular function after ultra-endurance exercise. Heart 2008;94:860-6

3. Naylor LH, George K, O'Driscoll G, et al. The athlete's heart: a contemporary appraisal of the 'Morganroth hypothesis'. Sports Med 2008;38:69-90.

4. Shave R, Baggish A, George K, et al. Exercise-induced cardiac troponin elevation: evidence, mechanisms and implications. J Am Coll Cardiol 2010;56:169-76.

5. George K, Spence A, Naylor LH, et al. Cardiac adaptation to acute and chronic participation in endurance sports. Heart 2011;97:1999-2004.

6. George KP, Wolfe LA, Burggraf GW. The 'athletic heart syndrome'. A critical review. Sports Med 1991:11:300-30. 
7. Morganroth J, Maron BJ, Henry WL, et al. Comparative left ventricular dimensions in trained athletes. Ann Intern Med 1975;82:521-4.

8. Longhurst JC, Kelly AR, Gonyea WJ, et al. Echocardiographic left ventricular masses in distance runners and weight lifters. J Appl Physiol 1980;48:154-62.

9. Baggish AL, Wang F, Weiner RB, et al. Training-specific changes in cardiac structure and function: a prospective and longitudinal assessment of competitive athletes. J Appl Physiol 2008;104:1121-8.

10. Mitchell JH, Haskell WL, Raven PB. Classification of sports. Med Sci Sport Exerc 1994;26:S242-5

11. Haykowsky MJ, Quinney HA, Gillis R, et al. Left ventricular morphology in junior and master resistance trained athletes. Med Sci Sports Exerc 2000;32:349-52.

12. Kinoshita N, Onishi S, Yamamoto S, et al. Unusual left ventricular dilatation without functional or biochemical impairment in normotensive extremely overweight Japanese professional sumo wrestlers. Am J Cardiol 2003;91:699-703.

13. Haykowsky M, Humen D, Teo $\mathrm{K}$, et al. Effects of 16 weeks of resistance training on left ventricular morphology and systolic function in healthy men $>60$ years of age. Am J Cardiol 2000;85:1002-6.

14. Spence AL, Naylor $\mathrm{LH}$, Carter $\mathrm{HH}$, et al. A prospective randomised longitudinal MR study of left ventricular adaptation to endurance and resistance exercise training in humans. J Physiol 2011;589:5443-52.

15. Pluim BM, Zwinderman AH, van der Laarse A, et al. The athlete's heart. A meta-analysis of cardiac structure and function. Circulation 2000;101:336-44.

16. Whyte GP, George K, Nevill A, et al. Left ventricular morphology and function in female athletes: a meta-analysis. Int J Sports Med 2004;25:380-3.

17. Pelliccia A, Maron BJ, Spataro A, et al. The upper limit of physiologic cardiac hypertrophy in highly trained elite athletes. N Engl J Med 1991;324:295-301.

18. Caselli S, Di Paolo FM, Pisicchio C, et al. Three-dimensional echocardiographic characterization of left ventricular remodeling in Olympic athletes. Am J Cardiol 2011;108:141-7.

19. Nagashima J, Musha $\mathrm{H}$, Takada $\mathrm{H}$, et al. New upper limit of physiologic cardiac hypertrophy in Japanese participants in the 100-km ultramarathon. J Am Coll Cardiol 2003;42:1617-23.

20. Rodriguez Reguero JJ, Iglesias Cubero G, Lòpez de la Iglesia J, et al. Prevalence and upper limit of cardiac hypertrophy in professional cyclists. Eur J Appl Physiol Occup Physiol 1995; 70:375-8.

21. George KP, Warburton DE, Oxborough D, et al. Upper limits of physiological cardiac adaptation in ultramarathon runners. J Am Coll Cardiol 2011;57:754-5.

22. Hoogsteen J, Hoogeveen A, Schaffers $H$, et al. Myocardial adaptation in different endurance sports: an echocardiographic study. Int J Cardiovasc Imaging 2004;20:19-26.

23. Abergel E, Chatellier G, Hagege AA, et al. Serial left ventricular adaptations in world-class cyclists. J Am Coll Cardiol 2004;44:144-9.

24. La Gerche A, Burns AT, D'hooge J, et al. Exercise strain rate imaging demonstrates normal right ventricular contractile reserve and clarifies ambiguous resting measures in endurance athletes. J Am Soc Echocardiogr 2012;25:253-62.

25. Oxborough D, Sharma $S$, Shave $R$, et al. The right ventricle of the endurance athlete: the relationship between morphology and deformation. J Am Soc Echocardiogr 2012;25:263-71.

26. D'Andrea A, Riegler L, Golia E, et al. Range of right heart measurements in top-level athletes: the training impact. Int J Cardiol 2011;July 5: epub ahead of print.

27. Wilhelm M, Roten $\mathrm{L}$, Tanner $\mathrm{H}$, et al. Atrial remodeling, autonomic tone and lifetime training hours in non-elite athletes. Am J Cardiol 2011;108:580-5.

28. Maron BJ, Epstein SE, Roberts WC. Causes of sudden death in competitive athletes. J Am Coll Cardiol 1986;7:204-14.

29. Maron BJ, Thompson PD, Ackerman MJ, et al. Recommendations and considerations related to preparticipation screening for cardiovascular abnormalities in competitive athletes: 2007 update: a scientific statement from the American Heart Association Council on nutrition, physical activity, and metabolism: endorsed by the American College of Cardiology Foundation. Circulation 2007:115:1643-55.

30. Wilson MG, Chandra N, Papadakis M, et al. Hypertrophic cardiomyopathy and ultra-endurance running - two incompatible entities? J Cardiovasc Magn Reson 2011;29:77.

31. Maron BJ, Wesley YE, Arce J. Hypertrophic cardiomyopathy compatible with successful completion of the marathon. Am J Cardiol 1984;53:1470-1.

32. Sharma S, Whyte G, Elliott P, et al. Electrocardiographic changes in 1000 highly trained junior elite athletes. Br J Sports Med 1999:33:319-24.

33. Corrado D, Pelliccia A, Heidbuchel $H$, et al. Recommendations for interpretation of 12-lead electrocardiogram in the athlete. Eur Heart J 2010;31:243-59.

34. Pelliccia A, Maron BJ, Culasso F, et al. Clinical significance of abnormal electrocardiographic patterns in trained athletes. Circulation 2000;102:278-84.

35. Corrado D, Pelliccia A, Bjornstad HH, et al. Cardiovascular pre-participation screening of young competitive athletes for prevention of sudden death: proposal fo a common European protocol. Consensus Statement of the Study Group of Sport Cardiology of the Working Group of Cardiac Rehabilitation and Exercise Physiology and the Working Group of Myocardial and Pericardial Diseases of the European Society of Cardiology. Eur Heart J 2005;26:516-24.
36. Pelliccia A, Di Paolo FM, Quattrini FM, et al. Outcomes in athletes with marked ECG repolarization abnormalities. N Engl J Med 2008;358:152-61.

37. Uberoi A, Stein R, Perez MV, et al. Interpretation of the electrocardiogram of young athletes. Circulation 2011;124:746-57.

38. Papadakis M, Carre F, Kervio G, et al. The prevalence, distribution and clinical outcomes of electrocardiographic replorization patterns in male athletes of African/ Afro-Caribbean origin. Eur Heart J 2011;32:2304-13

39. Rawlins J, Carre F, Kervio G, et al. Ethnic differences in physiological cardiac adaptation to intense physical exercise in highly trained female athletes. Circulation 2010:121:1078-85

40. De Bacquer D, De Backer G, Kornitzer M, et al. Prognostic value of ischemic electrocardiographic findings for cardiovascular mortality in men and women. J Am Coll Cardiol 1998;32:680-5.

41. Coronary artery surgery study (CASS). A randomized trial of coronary artery bypass surgery: survival data. Circulation 1983;68:939-50.

42. Rose G, Baxter PJ, Reid DD, et al. Prevalence and prognosis of electrocardiographic findings in middle-aged men. Br Heart J 1978;40:636-43.

43. Corrado D, Schmied C, Basso C, et al. Risk of sports: do we need a pre-participation screening for competitive and leisure athletes. Euro Heart $\mathrm{J}$ 2011;32:934-44.

44. Scharhag J, Schneider G, Urhausen A, et al. Athletes heart: right and left ventricular mass and function in male endurance athletes and untrained individuals determined by magnetic resonance imaging. J Am Coll Cardiol 2002;40:1856-63.

45. Prakken N, Velthuis B, Teske A, et al. Cardiac MRI reference values for athletes and nonathletes corrected for body surface area, training hours/week and sex. Eur $\mathrm{J}$ Cardiovasc Prev Rehabil 2010;17:198-203.

46. La Gerche A, Heidbuchel H, Burns AT, et al. Disproportionate exercise load and remodeling of the athlete's right ventricle. Med Sci Sports Exerc 2011b;43:974-81.

47. Yoerger D, Marcus F, Sherrill D, et al. Echocardiographic findings in patients meeting task force criteria arrhythmogenic right ventricular dysplasia. J Am Coll Cardiol 2005;45:860-5.

48. Marcus F, Towbin JA, Zareba W, et al. Arrhythmogenic right ventricular dysplasia/ cardiomyopathy (ARVD/C): a multidisciplinary study: design and protocol. Circulation 2003;107:2975-8

49. Bauce B, Frigo G, Benini G, et al. Differences and similarities between arrhythmogenic right ventricular cardiomyopathy and athlete's heart adaptations. $\mathrm{Br} \mathrm{J}$ Sports Med 2010;44:148-54

50. Rudski LG, Lai WW, Afilalo J, et al. Guidelines for the echocardiographic assessment of the right heart in adults: a report from the American Society of Echocardiography: endorsed by the European Association of Echocardiography, a registered branch of the European Society of Cardiology, and the Canadian Society of Echocardiography. J Am Soc Echocardiogr 2010;23:685-713

51. Teske AJ, Prakken NH, De Boeck BW, et al. Echocardiographic tissue deformation imaging of right ventricular systolic function in endurance athletes. Eur Heart $J$ 2009:30:969-77.

52. Mont L, Sambola A, Brugada J, et al. Long-lasting sport practice and lone atrial fibrillation. Eur Heart J 2002;23:477-82.

53. Molina L, Mont L, Marrugat J, et al. Long-term endurance sport practice increases the incidence of lone atrial fibrillation in men: a follow-up study. Europace 2008;10:618-23.

54. Lang RM, Bierig M, Devereux RB, et al. Recommendations for chamber quantification: a report from the American Society of Echocardiography's Guidelines and Standards Committee and the Chamber Quantification Writing Group, developed in conjunction with the European Association of Echocardiography, a branch of the European Society of Cardiology. J Am Soc Echocardiogr 2005;18:1440-63.

55. Mont L, Elosua R, Brugada J. Endurance sport practice as a risk factor for atria fibrillation and atrial flutter. Europace 2009;11:11-17

56. D'Andrea A, De Corato G, Scarafile R, et al. Left atrial myocardial function in either physiological or pathological left ventricular hypertrophy: a two-dimensional speckle strain study. Br J Sports Med 2008;42:696-702

57. Dawson $\mathbf{E}$, George K, Shave R, et al. Does the human heart fatigue subsequent to prolonged exercise? Sports Med 2003;33:365-80.

58. Saltin B, Stenberg J. Circulatory response to prolonged severe exercise. J App/ Physiol 1964;19:833-8.

59. Middleton N, Shave R, George K, et al. Left ventricular function immediately following prolonged exercise: a meta-analysis. Med Sci Sports Exerc 2006; 38:681-7.

60. Shave R, George K, Whyte G, et al. Postexercise changes in left ventricular function: the evidence so far. Med Sci Sports Exerc 2008:40:1393-9.

61. Oxborough D, Birch K, Shave R, et al. Exercise-induced cardiac fatigue: a review of the echocardiographic literature. Echocardiography 2010;27:1130-40.

62. McGavock JM, Warburton DE, Taylor D, et al. The effects of prolonged strenuous exercise on left ventricular function: a brief review. Heart Lung 2002;31:279-92.

63. Oxborough D, Shave R, Warburton $\mathrm{D}$, et al. Dilatation and dysfunction of the right ventricle immediately after ultraendurance exercise: exploratory insights from conventional two-dimensional and speckle tracking echocardiography. Circulation: Cardiovasc Imaging 2011;4:253-63. 
64. La Gerche A, Burns AT, Mooney DJ, et al. Exercise-induced right ventricular dysfunction and structural remodelling in endurance athletes. Eur Heart $\mathrm{J}$ 2012;33:998-1006.

65. Oxborough D, Whyte G, Wilson $\mathrm{M}$, et al. A depression in left ventricular diastolic filling following prolonged strenuous exercise is associated with changes in left atrial mechanics. J Am Soc Echocardiogr 2010;23:968-76.

66. Scott JM, Warburton DE. Mechanisms underpinning exercise-induced changes in left ventricular function. Med Sci Sports Exerc 2008;40:1400-7.

67. Welsh RC, Warburton DE, Humen DP, et al. Prolonged strenuous exercise alters the cardiovascular response to dobutamine stimulation in male athletes. J Physiol 2005;569:325-30.

68. Hart E, Dawson E, Rasmussen $\mathrm{P}$, et al. Beta-adrenergic receptor desensitization in man: insight into post-exercise attenuation of cardiac function. J Physiol 2006:577:717-25.

69. Vitiello D, Boissière J, Doucende G, et al. $\beta$-Adrenergic receptors desensitization is not involved in exercise-induced cardiac fatigue: NADPH oxidase-induced oxidative stress as a new trigger. J Appl Physiol 2011;111:1242-8.

70. Chan-Dewar F, Oxborough D, Shave R, et al. Evidence of increased electro-mechanical delay in the left and right ventricle after prolonged exercise. Eur J Appl Physiol 2010;108:581-7.

71. Chan-Dewar F, Gregson W, Whyte G, et al. Cardiac electromechanical delay is increased during recovery from $40 \mathrm{~km}$ cycling but is not mediated by exercise intensity. Scand J Med Sci Sports 2011 Sep 13. doi: 10.1111/j.1600-0838.2011. 01376.x [Epub ahead of print].

72. Neilan TG, Yoerger DM, Douglas PS, et al. Persistent and reversible cardiac dysfunction among amateur marathon runners. Eur Heart J 2006;27:1079-84.

73. Heidbuchel H, Hoogsteen J, Fagard R, et al. High prevalence of right ventricular involvement in endurance athletes with ventricular arrhythmias: role of an electrophysiological study in risk stratification. Eur Heart J 2003;24:1473-80.

74. Ector J, Ganame J, van der Merwe N, et al. Reduced right ventricular ejection fraction in endurance athletes presenting with ventricular arrhythmias: a quantitative angiographic assessment. Eur Heart J 2007;28:345-53.

75. Whyte GP. Clinical significance of cardiac damage and changes in function after exercise. Med Sci Sports Exerc 2008;40:1416-23.

76. Scharhag J, George K, Shave R, et al. Exercise-associated increases in cardiac biomarkers. Med Sci Sports Exerc 2008:40:1408-15.

77. Shave R, George KP, Atkinson G, et al. Exercise-induced cardiac troponin T release: a meta-analysis. Med Sci Sports Exerc 2007;39:2099-106.

78. Sahlen A, Gustafsson TP, Svensson JE, et al. Predisposing factors and consequences of elevated biomarker levels in long-distance runners aged $>55$ years. Am J of Cardiol 2009;104:1434-40.

79. Mehta R, Gaze D, Mohan S, et al. Post-exercise cardiac troponin release is related to exercise training history. Int J Sports Med 2012;33:333-7.
80. Middleton N, George K, Whyte G, et al. Cardiac troponin T release is stimulated by endurance exercise in healthy humans. J Am Coll Cardiol 2008;52:1813-14.

81. Shave R, Ross P, Low D, et al. Cardiac troponin I is released following high-intensity short-duration exercise in healthy humans. Int J Cardiol 2010b;145:337-9.

82. Lee IM, Manson JE, Hennekens $\mathrm{CH}$, et al. Body weight and mortality: a 27-year follow-up of middle-aged men. JAMA 1993;270:2823-8.

83. Grimsmo J, Maehlum S, Moelstad P, et al. Mortality and cardiovascular morbidity among long-term endurance male cross country skiers followed for 28-30 years. Scand J Med Sci Sports 2011;21:e351-8.

84. Maron BJ, Pelliccia A, Spataro A, et al. Reduction in left ventricular wall thickness after deconditioning in highly trained Olympic athletes. Br J Sports Med 1993;69:125-8

85. Pelliccia A, Kinoshita n, Pisicchio $C$, et al. Long-term clinical consequences of intense, uninterrupted endurance training in Olympic athletes. J Am Coll Cardiol 2010;55:1619-25.

86. Pelliccia A, Maron BJ, De Luca R, et al. Remodeling of left ventricular hypertrophy in elite athletes after long-term deconditioning. Circulation 2002;105:944-9.

87. Wilson M, O'Hanlon R, Prasad S, et al. Myocardial fibrosis in an veteran endurance athlete. BMJ Case Rep 2009;piibcr121345.

88. Whyte G, Sheppard M, George K, et al. Post-mortem evidence of idiopathic left ventricular hypertrophy and idiopathic interstitial myocardial fibrosis: is exercise the cause? BMJ Case Rep 2009;piibcr0820080758

89. Benito B, Gay-Jordi G, Serrano-Mollar A, et al. Cardiac arrhythmogenic remodeling in a rat model of long-term intensive exercise training/clinical perspective. Circulation 2011:123:13-22.

90. Breuckmann F, Möhlenkamp S, Nassenstein K, et al. Myocardial late gadolinium enhancement: prevalence, pattern, and prognostic relevance in marathon runners. Radiology 2009;251:50-7.

91. Wilson MG, O'Hanlon R, Prasad S, et al. Diverse patterns of myocardial fibrosis in lifelong, veteran endurance athletes. J Appl Physiol 2011;110:1622-6.

92. Abdulla J, Nielsen JR. Is the risk of atrial fibrillation higher in athletes than in the general population? A systematic review and meta-analysis. Europace 2009;11:1156-9.

93. Claessen G, Colyn E, La Gerche A, et al. Long-term endurance sport is a risk factor for development of lone atrial flutter. Heart 2011;97:918-22.

94. Biffi A, Maron BJ, Culasso F, et al. Patterns of ventricular tachyarrhythmias associated with training, deconditioning and retraining in elite athletes without cardiovascular abnormalities. Am J Cardiol 2011;107:697-703.

95. Biffi A, Maron BJ, Di Giacinto, et al. Relations between training-induced left ventricular hypertrophy and risk for ventricular tachyarrhythmias in elite athletes. $\mathrm{Am}$ J Cardiol 2008;101:1792-5.

96. Biffi A, Ansalone G, Verdile L, et al. Ventricular arrhythmias and athletes heart: role of signal-averaged electrocardiography. Eur Heart J 1996:17:557-63. 